\title{
On general partial Gaussian sums
}

\section{Ganglian Ren ${ }^{1}$, Dingding $\mathrm{He}^{2}$ and Tianping Zhang ${ }^{3^{*}}$}

"Correspondence:

tpzhang@snnu.edu.cn

${ }^{3}$ School of Mathematics and Information Science, Shaanxi

Normal University, Xi'an, Shaanxi

710119, P.R. China

Full list of author information is

available at the end of the article

\section{Abstract \\ Let $q \geq 2$ be a fixed integer, $A=A(q) \leq q, B=B(q) \leq q$, and $H=H(q) \leq q$. Define

$$
\hbar(A, B, H)=\{a \in \mathbb{Z}|(a, q)=1, a b \equiv 1 \quad(\bmod q), 1 \leq a \leq A, 1 \leq b \leq B,| a-b \mid \leq H\} .
$$

With the aid of the estimates for the general Kloosterman sums and the properties of trigonometric sums, we obtain an upper bound of the general partial Gaussian sums over the number set $\hbar(A, B, H)$.

MSC: $11 \mathrm{~L} 05 ; 11 \mathrm{~L} 40$

Keywords: partial Gaussian sums; Kloosterman sums

\section{Introduction}

Let $q, N, H, n$ be integers with $q \geq 2, H>0, \chi$ be a Dirichlet character $\bmod q$ and $e(y)=$ $e^{2 \pi i y}$. The study of the following partial Gaussian sums:

$$
\sum_{a=N+1}^{N+H} \chi(a) e\left(\frac{n a}{q}\right)
$$

is of great importance. By extending his well-known work on character sums, Burgess obtained the following.

Proposition 1 ([1]) Let $q$ be a prime and $\chi$ be a non-principal Dirichlet character mod $q$. Then, for any integers $N, n, H, r$ with $0<H<q$ and $r \geq 2$, we have

$$
\sum_{a=N+1}^{N+H} \chi(a) e\left(\frac{n a}{q}\right) \ll H^{1-1 / r} q^{1 / 4(r-1)} \log ^{2} q .
$$

Proposition 2 ([2]) Let $q \geq 2$ be an integer and $\chi$ be a primitive character mod $q$. Then, for any integers $N, n, H$ with $0<H$, we have

$$
\sum_{a=N+1}^{N+H} \chi(a) e\left(\frac{n a}{q}\right) \ll H^{2 / 3} q^{1 / 8+\epsilon} .
$$


Proposition 3 ([3]) Let $q=p^{\alpha}(\alpha>1)$ be a power of the prime $p>3$ and $\chi$ be a nonprincipal Dirichlet character mod $q$. Then, for any integers $N, n, H$ with $0<H$, we have

$$
\sum_{a=N+1}^{N+H} \chi(a) e\left(\frac{n a}{q}\right) \ll H^{3 / 4} q^{1 / 12} \log ^{3} q
$$

At almost the same time, Liu [4] showed independently the following.

Proposition 4 Let $q$ be a prime power, $\chi, \psi$ be a multiplicative and additive character mod q, respectively, with $\chi$ non-principal. Then, for any integers $N, H$ with $0<H$, we have

$$
\sum_{a=N+1}^{N+H} \chi(a) \psi(a) \ll H^{3 / 4} q^{1 / 12+\epsilon} .
$$

Now let $q \geq 2$ be a fixed integer, $A=A(q) \leq q, B=B(q) \leq q$, and $H=H(q) \leq q$. Define

$$
\hbar(A, B, H)=\{a \in \mathbb{Z}|(a, q)=1, a b \equiv 1 \quad(\bmod q), 1 \leq a \leq A, 1 \leq b \leq B,| a-b \mid \leq H\} .
$$

It is a direct generalization of the set of so-called $H$-flat numbers $\bmod q$, which was studied extensively by $\mathrm{Xi}$ (see [5] and references therein).

This paper deals with general partial Gaussian sums of the following type:

$$
G_{k}(\chi, A, B, H ; q)=\sum_{a \in \hbar(A, B, H)} a^{k} \chi(a) e\left(\frac{n a}{q}\right),
$$

where $k \geq 0$ is an arbitrary fixed integer. For the sake of periodicity of $e\left(\frac{n a}{q}\right)$ we can also restrict $n$ to be $1 \leq n \leq q$. Then with the aid of the estimates for the general Kloosterman sums and the properties of trigonometric sums, we shall obtain upper bound estimates as follows.

Theorem Let $q \geq 2$ be an integer and $\chi$ a non-principal Dirichlet character mod $q$. Then

$$
G_{k}(\chi, A, B, H ; q) \ll A^{k} q^{1 / 2} d(q)\left(\frac{n A B d(q)}{q^{2}}+\frac{B d(q)(\log q)(\log H)}{q}+\log ^{3} q\right),
$$

which is uniformly nontrivial for any positive integer $n$ such that $n<q^{1 / 2}$.

Taking $n$ a constant, we can immediately obtain the following.

Corollary 1 Let $q \geq 2$ be an integer and $\chi$ a non-principal Dirichlet character mod $q$. Then we have

$$
G_{k}(\chi, A, B, H ; q) \ll A^{k} q^{1 / 2} d(q)\left(\frac{A B d(q)}{q^{2}}+\frac{B d(q)(\log q)(\log H)}{q}+\log ^{3} q\right) .
$$

Taking $k=0, B=q$ in Corollary 1 , we obtain the following. 
Corollary 2 Let $q \geq 2$ be an integer and $\chi$ a non-principal Dirichlet character mod $q$. Then, for any positive integers $A, H$ such that $A, H \leq q$, we have

$$
G_{0}(\chi, A, q, H ; q) \ll q^{1 / 2} d^{2}(q) \log ^{3} q .
$$

Remark It is easy to see that (1.3) is stronger than (1.1) for any integer $H$ such that $q^{9 / 16+\epsilon}<$ $H \leq q$. It is also stronger than (1.2) for any integer $H$ such that $q^{5 / 9} d^{8 / 3}(q)<H \leq q$. These results reveal that more cancelations occurred in the number set $\hbar(A, B, H)$.

Taking $A=H=q$ in Corollary 2, we obtain the following.

Corollary 3 Let $q \geq 2$ be an integer and $\chi$ a non-principal Dirichlet character mod $q$. Then we have

$$
G_{0}(\chi, q, q, q ; q)=\sum_{a=1}^{q} \chi(a) e\left(\frac{n a}{q}\right) \ll q^{1 / 2} d^{2}(q) \log ^{3} q .
$$

This is a little stronger than the classical result of the complete Gauss sums in the case when $(n, q)>1$.

\section{Some lemmas}

To prove the theorem, we need the following lemmas.

Lemma 1 Let $q, n, \ell, r$ be integers with $q>2, q>n$, and $\ell \geq 0$. Define $h(r, \ell ; n)=$ $\sum_{a=1}^{n} a^{\ell} e\left(\frac{r a}{q}\right)$. Then we have

$$
h(r, \ell ; n) \begin{cases}=\frac{n^{\ell+1}}{\ell+1}+O\left(n^{\ell}\right), & q \mid r, \\ \ll \frac{n^{\ell}}{|\sin (\pi s / q)|}, & q \nmid r,\end{cases}
$$

where $s=\min (r, q-r)$ with $1 \leq r \leq q-1$.

Proof See Lemma 3 of [6] or Lemma 2.4 of [7].

Lemma 2 Let $q$ be a positive integer. Then we have

$$
\left|K_{\chi}(m, n ; q)\right| \leq q^{1 / 2}(m, n, q)^{1 / 2} d(q),
$$

where $K_{\chi}(m, n ; q)=\sum_{a(\bmod q)} \chi(a) e\left(\frac{m a+n \bar{a}}{q}\right)$ is the general Kloosterman sum, with a $\bar{a} \equiv 1$ $(\bmod q)$ and $(m, n, q)$ the greatest common divisor of $m, n, q$.

Proof See Lemma 1 of [5].

\section{Proof of the Theorem}

Now we come to prove the theorem. Note that

$$
G_{k}(\chi, A, B, H ; q)=\sum_{t \leq H} \sum_{\substack{a \leq A, b \leq B \\ a-b \equiv t \quad(\bmod q), a b \equiv 1}} a^{k} \chi(a) e\left(\frac{n a}{q}\right) .
$$


Applying the trigonometric sums identity

$$
\sum_{a=1}^{q} e\left(\frac{m a}{q}\right)= \begin{cases}q, & q \mid m, \\ 0, & q \nmid m,\end{cases}
$$

we obtain

$$
\begin{aligned}
& G_{k}(\chi, A, B, H ; q) \\
& =\sum_{a \in \hbar(A, B, H)} a^{k} \chi(a) e\left(\frac{n a}{q}\right) \\
& =\sum_{t \leq H} \sum_{\substack{a \leq A, b \leq B \\
a-b \equiv t}}^{\prime} a^{k} \chi(a) e\left(\frac{n a}{q}\right) \\
& =\frac{1}{q} \sum_{m \leq q} \sum_{t \leq H} e\left(-\frac{m t}{q}\right) \sum_{\substack{a \leq A, b \leq B \\
a b \equiv 1(\bmod q)}}^{\prime} a^{k} \chi(a) e\left(\frac{(m+n) a-m b}{q}\right) \\
& =\frac{1}{q^{3}} \sum_{m \leq q} \sum_{t \leq H} e\left(-\frac{m t}{q}\right) \sum_{\substack{a, b \leq q \\
a b \equiv 1}}^{\prime(\bmod q)} \chi(a) e\left(\frac{(m+n) a-m b}{q}\right) \\
& \times \sum_{c \leq A} c^{k} \sum_{r \leq q} e\left(\frac{r(a-c)}{q}\right) \sum_{d \leq B} \sum_{s \leq q} e\left(\frac{s(b-d)}{q}\right) \\
& =\frac{1}{q^{3}} \sum_{r, s \leq q} \sum_{m \leq q} \sum_{t \leq H} e\left(-\frac{m t}{q}\right) \sum_{\substack{a, b \leq q \\
a b \equiv 1}}^{\prime} \chi(a) e\left(\frac{(m+r+n) a-(m-s) b}{q}\right) \\
& \times \sum_{c \leq A} c^{k} e\left(-\frac{r c}{q}\right) \sum_{d \leq B} e\left(-\frac{s d}{q}\right) \\
& =\frac{1}{q^{3}} \sum_{r, s \leq q} \sum_{m \leq q} \sum_{t \leq H} e\left(-\frac{m t}{q}\right) K_{\chi}(m+r+n, s-m ; q) h(-r, k ; A) h(-s, 0 ; B) \\
& =\frac{1}{q^{3}} \sum_{m \leq q} \sum_{t \leq H} e\left(-\frac{m t}{q}\right) K_{\chi}(m+n,-m ; q) h(-q, k ; A) h(-q, 0 ; B) \\
& +\frac{1}{q^{3}} \sum_{r \leq q-1} \sum_{m \leq q} \sum_{t \leq H} e\left(-\frac{m t}{q}\right) K_{\chi}(m+r+n,-m ; q) h(-r, k ; A) h(-q, 0 ; B) \\
& +\frac{1}{q^{3}} \sum_{s \leq q-1} \sum_{m \leq q} \sum_{t \leq H} e\left(-\frac{m t}{q}\right) K_{\chi}(m+n, s-m ; q) h(-q, k ; A) h(-s, 0 ; B) \\
& +\frac{1}{q^{3}} \sum_{r, s \leq q-1} \sum_{m \leq q} \sum_{t \leq H} e\left(-\frac{m t}{q}\right) K_{\chi}(m+r+n, s-m ; q) h(-r, k ; A) h(-s, 0 ; B) \text {. }
\end{aligned}
$$

Then from Lemma 1 and Lemma 2, we have

$$
\begin{aligned}
\frac{1}{q^{3}} & \sum_{m \leq q} \sum_{t \leq H} e\left(-\frac{m t}{q}\right) K_{\chi}(m+n,-m ; q) h(-q, k ; A) h(-q, 0 ; B) \\
& \ll \frac{1}{q^{3}} \sum_{m \leq q} \min \left(H,\left\|\frac{m}{q}\right\|^{-1}\right)\left|K_{\chi}(m+n,-m ; q)\right| \cdot|h(-q, k ; A)| \cdot|h(-q, 0 ; B)|
\end{aligned}
$$




$$
\begin{aligned}
& \ll H A^{k+1} B q^{-5 / 2} d(q) \sum_{m \leq q / H}(m+n, q)^{1 / 2} \\
& +A^{k+1} B q^{-3 / 2} d(q) \sum_{q / H<m \leq q-1} \frac{(m+n, q)^{1 / 2}}{m},
\end{aligned}
$$

where $\|x\|=\min _{a \in Z}|x-a|$.

Combining the estimates

$$
\begin{aligned}
\sum_{m \leq q / H}(m+n, q)^{1 / 2} & =\sum_{d \mid q} d^{1 / 2} \sum_{\substack{m \leq q / H \\
d \mid(m+n)}} 1 \\
& =\sum_{d \mid q} d^{1 / 2} \sum_{m \leq q /(d H)+n / d} 1 \\
& \ll H^{-1} q d(q)+n d(q)
\end{aligned}
$$

and

$$
\begin{aligned}
\sum_{q / H<m \leq q-1} \frac{(m+n, q)^{1 / 2}}{m} & =\sum_{d \mid q} d^{1 / 2} \sum_{\substack{q / H<m \leq q-1 \\
d \mid(m+n)}} \frac{1}{m} \\
& =\sum_{d \mid q} d^{1 / 2} \sum_{\frac{q}{H d}+\frac{n}{d}<m \leq \frac{q-1+n}{d}} \frac{1}{d m-n} \\
& \ll \sum_{d \mid q} d^{-1 / 2} \sum_{\frac{q}{H d}+\frac{n}{d}<m \leq \frac{q-1+n}{d}} \frac{1}{m}\left(1+\frac{n}{d m}\right) \\
& \ll d(q) \log H+n d(q),
\end{aligned}
$$

we have

$$
\begin{aligned}
\frac{1}{q^{3}} & \sum_{m \leq q} \sum_{t \leq H} e\left(-\frac{m t}{q}\right) K_{\chi}(m+n,-m ; q) h(-q, k ; A) h(-q, 0 ; B) \\
& \ll n A^{k+1} B q^{-3 / 2} d^{2}(q)+A^{k+1} B q^{-3 / 2} d^{2}(q) \log H .
\end{aligned}
$$

Applying Lemma 1 and Lemma 2 again, we obtain

$$
\begin{aligned}
& \frac{1}{q^{3}} \sum_{r \leq q-1} \sum_{m \leq q} \sum_{t \leq H} e\left(-\frac{m t}{q}\right) K_{\chi}(m+r+n,-m ; q) h(-r, k ; A) h(-q, 0 ; B) \\
& \quad \ll \frac{1}{q^{3}} \sum_{r \leq q-1} \sum_{m \leq q} \min \left(H,\left\|\frac{m}{q}\right\|^{-1}\right)\left|K_{\chi}(m+r+n,-m ; q)\right| \cdot|h(-r, k ; A)| \cdot|h(-q, 0 ; B)| \\
& \ll H B q^{-5 / 2} d(q) \sum_{r \leq q-1} \sum_{m \leq q / H}(m+r+n,-m, q)^{1 / 2} \cdot \frac{A^{k}}{\left|\sin \left(\frac{\pi r}{q}\right)\right|} \\
& \quad+B q^{-3 / 2} d(q) \sum_{r \leq q-1} \sum_{q / H<m \leq q-1} \frac{(m+r+n,-m, q)^{1 / 2}}{m} \cdot \frac{A^{k}}{\left|\sin \left(\frac{\pi r}{q}\right)\right|}
\end{aligned}
$$




$$
\begin{aligned}
\ll & H A^{k} B q^{-3 / 2} d(q) \sum_{r \leq q-1} \frac{1}{r} \sum_{m \leq q / H}(m+r+n,-m, q)^{1 / 2} \\
& +A^{k} B q^{-1 / 2} d(q) \sum_{r \leq q-1} \frac{1}{r} \sum_{q / H<m \leq q-1} \frac{(m+r+n,-m, q)^{1 / 2}}{m} .
\end{aligned}
$$

Combining

$$
\begin{aligned}
& \sum_{r \leq q-1} \frac{1}{r} \sum_{m \leq q \mid H}(m+r+n,-m, q)^{1 / 2} \\
& =\sum_{d \mid q} d^{1 / 2} \sum_{\substack{r \leq q \\
d \mid(r+n)}} \frac{1}{r} \sum_{\substack{m \leq q / H \\
d \mid m}} 1 \\
& =\sum_{d \mid q} d^{1 / 2} \sum_{(n+1) / d \leq r \leq(q+n-1) / d} \frac{1}{d r-n} \sum_{m \leq q /(H d)} 1 \\
& \ll q / H \sum_{d \mid q} d^{-3 / 2} \sum_{(n+1) / d \leq r \leq(q+n-1) / d} \frac{1}{r}\left(1+\frac{n}{d r}\right) \\
& \ll H^{-1} q d(q) \log \left(\frac{q+n-1}{n+1}\right)
\end{aligned}
$$

and

$$
\begin{aligned}
& \sum_{r \leq q-1} \frac{1}{r} \sum_{q / H<m \leq q-1} \frac{(m+r+n,-m, q)^{1 / 2}}{m} \\
& =\sum_{d \mid q} d^{1 / 2} \sum_{\substack{r \leq q-1 \\
d \mid(r+n)}} \frac{1}{r} \sum_{\substack{q / H<m \leq q-1 \\
d \mid m}} \frac{1}{m} \\
& =\sum_{d \mid q} d^{-1 / 2} \sum_{(n+1) / d \leq r \leq(q+n-1) / d} \frac{1}{d r-n} \sum_{q /(H d)<m \leq q / d} \frac{1}{m} \\
& \ll(\log H) \sum_{d \mid q} d^{-3 / 2} \sum_{r \leq q / d} \frac{1}{r\left(1-\frac{n}{d r}\right)} \\
& \ll d(q)(\log H) \log \left(\frac{q+n-1}{n+1}\right),
\end{aligned}
$$

we have

$$
\begin{aligned}
\frac{1}{q^{3}} & \sum_{r \leq q-1} \sum_{m \leq q} \sum_{t \leq H} e\left(-\frac{m t}{q}\right) K_{\chi}(m+r+1,-m ; q) h(-r, k ; A) h(-q, 0 ; B) \\
& \ll A^{k} B q^{-1 / 2} d^{2}(q)(\log H) \log \left(\frac{q+n-1}{n+1}\right) .
\end{aligned}
$$

Similarly, we get the estimate

$$
\begin{aligned}
\frac{1}{q^{3}} & \sum_{s \leq q} \sum_{m \leq q} \sum_{t \leq H} e\left(-\frac{m t}{q}\right) K_{\chi}(m+1, s-m ; q) h(-q, k ; A) h(-s, 0 ; B) \\
& \ll A^{k+1} q^{-3 / 2} d^{2}(q) \log \left(\frac{q-1}{n+1}\right) .
\end{aligned}
$$


Noting that

$$
\begin{aligned}
& \frac{1}{q^{3}} \sum_{r, s \leq q-1} \sum_{m \leq q} \sum_{t \leq H} e\left(-\frac{m t}{q}\right) K_{\chi}(m+r+n, s-m ; q) h(-r, k ; A) h(-s, 0 ; B) \\
& \ll q^{-5 / 2} \tau(q) \sum_{r, s \leq q-1} \sum_{m \leq q} \min \left(H,\left\|\frac{m}{q}\right\|^{-1}\right) \cdot(m+r+n, s-m, q)^{1 / 2} \frac{A^{k}}{\left|\sin \left(\frac{\pi r}{q}\right)\right|} \cdot \frac{1}{\left|\sin \left(\frac{\pi s}{q}\right)\right|} \\
& \ll H A^{k} q^{-1 / 2} d(q) \sum_{r, s \leq q-1} \frac{1}{r s} \sum_{m \leq q / H}(m+r+n, s-m, q)^{1 / 2} \\
& \quad+A^{k} q^{1 / 2} d(q) \sum_{r, s \leq q-1} \frac{1}{r s} \sum_{q / H<m \leq q-1} \frac{(m+r+n, s-m, q)^{1 / 2}}{m} .
\end{aligned}
$$

Using the estimates

$$
\begin{aligned}
& \sum_{r, s \leq q-1} \frac{1}{r s} \sum_{m \leq q / H}(m+r+n, s-m, q)^{1 / 2} \\
& =\sum_{d \mid q} d^{1 / 2} \sum_{m \leq q / H} \sum_{\substack{r \leq q-1 \\
d \mid(m+r+n)}} \frac{1}{r} \sum_{\substack{s \leq q-1 \\
d \mid(s-m)}} \frac{1}{s} \\
& =\sum_{d \mid q} d^{1 / 2} \sum_{m \leq q / H(m+n+1) / d \leq r \leq(q+m+n-1) / d} \frac{1}{d r-m-n} \sum_{(1-m) / d \leq s \leq(q-m-1) / d} \frac{1}{d s+m} \\
& \ll H^{-1} q d(q)(\log q) \log (2 q+n-1)
\end{aligned}
$$

and

$$
\begin{aligned}
& \sum_{r, s \leq q-1} \frac{1}{r s} \sum_{q / H<m \leq q-1} \frac{(m+r+n, s-m, q)^{1 / 2}}{m} \\
& \quad=\sum_{d \mid q} d^{1 / 2} \sum_{q / H<m \leq q-1} \frac{1}{m} \sum_{\substack{r \leq q-1 \\
d \mid(m+r+n)}} \frac{1}{r} \sum_{\substack{s \leq q-1 \\
d \mid(s-m)}} \frac{1}{s} \\
& =\sum_{d \mid q} d^{1 / 2} \sum_{q / H<m \leq q-1} \frac{1}{m} \sum_{(m+n+1) / d \leq r \leq(q+m+n-1) / d} \frac{1}{d r-m-n} \sum_{(1-m) / d \leq s \leq(q-m-1) / d} \frac{1}{d s+m} \\
& \ll d(q)\left(\log ^{2} q\right) \log (2 q+n-1),
\end{aligned}
$$

we have

$$
\begin{aligned}
& \frac{1}{q^{3}} \sum_{r, s \leq q-1} \sum_{m \leq q} \sum_{t \leq H} e\left(-\frac{m t}{q}\right) K_{\chi}(m+r+n, s-m ; q) h(-r, k ; A) h(-s, 0 ; B) \\
& \quad \ll A^{k} q^{1 / 2} d(q)\left(\log ^{2} q\right) \log (2 q+n-1) .
\end{aligned}
$$

Now combining (3.1)-(3.4), we have

$$
G_{k}(\chi, A, B, H ; q) \ll A^{k} q^{1 / 2} d(q)\left(\frac{n A B d(q)}{q^{2}}+\frac{B d(q)(\log q)(\log H)}{q}+\log ^{3} q\right) .
$$

This completes the proof of the theorem. 
Competing interests

The authors declare that they have no competing interests.

\section{Authors' contributions}

DH drafted the manuscript. GR and TZ participated in its design and coordination and helped to draft the manuscript. All authors read and approved the final manuscript.

\section{Author details}

${ }^{1}$ College of Mathematics and Information Science, Xianyang Normal University, Xianyang, Shaanxi 712000, P.R. China.

${ }^{2}$ School of Mathematics, Northwest University, Xi'an, Shaanxi 710127, P.R. China. ${ }^{3}$ School of Mathematics and Information Science, Shaanxi Normal University, Xi'an, Shaanxi 710119, P.R. China.

\section{Acknowledgements}

The article was supported by the National Natural Science Foundation of China (Grant Nos. 11201275, 11471258), the Research Fund for the Doctoral Program of Higher Education of China (Grant No. 20106101120001), the Natural Science Foundation of Shaanxi Province of China (Grant Nos. 2011JQ1010, 2016JM1017), the Scientific Research Program Funded by Shaanxi Provincial Education Department (Grant No. 15JK1794) and the Fundamental Research Funds for the Central Universities (Grant No. GK201503014). The authors want to show their great thanks to the anonymous referee for his/her helpful comments and suggestions.

Received: 2 April 2016 Accepted: 9 November 2016 Published online: 22 November 2016

\section{References}

1. Burgess, DA: Partial Gaussian sums. Bull. Lond. Math. Soc. 20(6), 589-592 (1988)

2. Burgess, DA: Partial Gaussian sums. II. Bull. Lond. Math. Soc. 21(2), 153-158 (1989)

3. Burgess, DA: Partial Gaussian sums. III. Glasg. Math. J. 34(2), 253-261 (1992)

4. Liu, CL: On incomplete Gaussian sums. Acta Math. Sin. New Ser. 12(2), 141-150 (1996)

5. $X i, P, Y i, Y$ : On character sums over flat numbers. J. Number Theory 130(5), 1234-1240 (2010)

6. Xu, ZF: Distribution of the difference of an integer and its $m$-th power mod $n$ over incomplete intervals. J. Number Theory 133(12), 4200-4223 (2013)

7. Xu, ZF, Zhang, TP: High-dimensional D.H. Lehmer problem over short intervals. Acta Math. Sin. Engl. Ser. 30(2), 213-228 (2014)

\section{Submit your manuscript to a SpringerOpen ${ }^{\circ}$ journal and benefit from:}

- Convenient online submission

Rigorous peer review

- Immediate publication on acceptance

- Open access: articles freely available online

- High visibility within the field

- Retaining the copyright to your article 